\title{
COMENTARIO EDITORIAL: ¿Es necesario regular la publicidad de medicamentos y tecnología sanitaria dirigida directamente a los consumidores?
}

\begin{abstract}
Resumen
A propósito del trabajo recientemente publicado por Kravitz y col.. el autor de este artículo editorial describe y discute la influencia que tiene la publicidad de medicamentos y tecnologías sanitarias en las solicitudes de los pacientes y sus familias, y en los comportamientos prescriptivos de los profesionales de la salud. Toma posición a favor de la regulación de la publicidad directa a los consumidores con el objetivo de que estos puedan tomar las mejores decisiones para el cuidado de su salud y no lo hagan sobre la base de la medicina basada en la "evidencia del marketing".
\end{abstract}

Silva C. ¿Es necesario regular la publicidad de medicamentos y/o tecnología sanitaria dirigida directamente a los consumidores? Evid. actual. práct. ambul; $2006 ; 9: 1-2$.

La publicidad directa al consumidor implica importantes intereses económicos a nivel mundial. Para tener una idea de la magnitud, mencionaremos que Merck invirtió en el 20012.500 millones de dólares en la promoción del polémico Vioxx: 35 millones más que lo gastado por Pepsi en el período ${ }^{1}$.

Existe evidencia de que las sugerencias o solicitudes de los pacientes ${ }^{2}$ sobre la indicación de exámenes complementarios y/o tratamientos influyen en las prescripciones de los médicos, lo que puede conducir a la medicalización de fenómenos normales ${ }^{3}$. Como ejemplo cotidiano de los primeros y según nuestra experiencia de trabajo local, podemos mencionar el bombardeo televisivo sobre métodos para la valoración de la densidad ósea en la postmenopausia, el control prostático, etc.; y respecto de los últimos, los suplementos vitamínicos, los protectores hepáticos, la aspirina, otros analgésicos, etc.

Este fenómeno ocurre frecuentemente en casos en que esa prescripción no es adecuada. Por otro lado, los pacientes y/o sus familiares pueden sentirse decepcionados ${ }^{4}$ si no se responde a tal solicitud, pudiendo esta negativa ser interpretada como un comportamiento del profesional de la salud destinado a reducir los costos de atención por presiones del eventual pagador de los servicios y deteriorar la relación médico paciente.

El trabajo de Kravitz y col ch $^{5}$ que se resume a continuación resulta interesante y novedoso en cuanto a su metodología, ya que, a diferencia de otras experiencias en esta área de la investigación que se habían basado en encuestas ${ }^{6}$, se vale de un modelo experimental a través de un "ensayo clínico" ciego. Sus autores habían publicado numerosos trabajos sobre la relación médico paciente y, en especial, sobre la respuesta de los profesionales de la salud a los pedidos de los pacientes, así como sobre la manera en que los médicos requieren de la confianza de los pacientes para obtener una respuesta exitosa en los tratamientos.

En su ya clásica revisión $n^{7}$ publicada en el año 2000 advertían sobre lo perjudicial de la publicidad dirigida al consumidor y recomendaban intervenir a través de regulaciones estatales con el objetivo de preservar la salud pública y utilizar racionalmente los recursos. Los resultados del estudio que hoy comentamos sugieren que la influencia de la solicitud del paciente parece aumentar cuando existe más incertidumbre sobre el tratamiento. Demuestra también que la publicidad puede ayudar a reducir la subprescripción en individuos que padecen depresión mayor, pero también contribuir a la sobreprescripción en cuadros en los que no se requiere tratamiento farmacológico.

Este trabajo aborda un tema polémico y que puede conducir a la toma de posiciones extremas en detrimento de la sensatez. La necesidad de lucro de la principal industria ligada a la salud, como lo es la farmacéutica, el incremento sostenido del gasto en medicamentos, el uso de genéricos y nuevos medicamentos sin un claro beneficio, y el derecho de los pacientes a la información y su mayor decisión en temas vinculados a su salud, son algunos de los problemas; pudiendo los prejuicios rápidamente invadir al lector.

En muchos casos, supuestas campañas de información, muchas de ellas bienintencionadas desde los organizadores, son una forma encubierta de publicidad para estimular el consumo de un medicamento o grupo de medicamentos (por ej. "la semana de la osteoporosis", "de la próstata" u otras patologías que aunque importantes individualmente, no tienen la prioridad sanitaria para este tipo de acciones)

Aunque la depresión constituye una enfermedad prevalente, cróni$\mathrm{ca}$, con una fuerte carga de discapacidad que irá en aumento en los próximos años y existen tratamientos probados, la publicidad de un fármaco crea la falsa creencia de que puede ser resuelta mágicamente con una "pastillita", desplazando otros tratamientos más adecuados, seguros o al menos complementarios.

No hay que olvidar que el objetivo de la publicidad es vender. La información al paciente y sus necesidades difícilmente pueda ser compatible con los intereses de la industria.

La publicidad genera expectativas poco realistas sobre los beneficios de la medicación y una demanda inapropiada de nuevos y más costosos medicamentos, no siempre mejores y en la mayoría de los casos, de incierta seguridad.

Esto ha llevado a que el rubro farmacéutico ocupe los horarios centrales de la televisión encontrando a cualquier personaje de la farándula recomendando antitusivos, analgésicos o antiácidos y estimulando un consumo desmedido e inadecuado de fármacos. Esta práctica no hará más que aumentar la popularidad de ciertas marcas en detrimento de informaciones esenciales sobre las diversas opciones de tratamiento.

Es interesante destacar que en muchos países se encuentra vedada la publicidad de medicamentos directa al consumidor. En Argentina, hace pocos años se abrió esta posibilidad a medicamentos de venta libre, bajo el argumento de que es un derecho de los ciudadanos contar con información sobre los productos que pueden elegir.

El "empoderamiento" (castellanizando la palabra inglesa "empowerment") de los pacientes es una tendencia irreversible y ampliamente aceptada como una manera de asegurar los derechos individuales y tender a una mayor simetría en la relación médico paciente. Este proceso requiere que los pacientes dispongan de información adaptada culturalmente para la toma de decisiones. Aclaramos que en EEUU, el país donde se realizó el estudio que comentamos, se encuentra permitida la publicidad de medicamentos que sólo se expenden bajo receta e incluso de los psicofármacos. En Argentina, la Resolución Ministerial 20/058 y la Disposición 4980/05 regulan la publicidad dirigida al público. Pese a la norma y la posibilidad de realizar denuncias "on line" en forma anónima, es frecuente observar como sistemáticamente se atribuye a ciertos alimentos propiedades curativas o preventivas (ej. algunos derivados lácteos) y como los laboratorios farmacéuticos insinúan que sus medicamentos de venta libre tienen propiedades o indicaciones alejadas de la realidad. Consideramos que no es lo mismo insinuar que si compra determinado celular una señorita hermosa enloquecerá por usted que inducir al consumo injustificado de fármacos. Los pacientes necesitan una mayor y mejor información sobre los medicamentos. Sin embargo, dicha información no debería proceder directamente de las compañías farmacéuticas ya que esta práctica no hará más que aumentar la popularidad de ciertas marcas en detrimento de informaciones esenciales sobre las diversas opciones de tratamiento.

No existe ninguna evidencia de beneficios en la población, los pacientes o los sistemas de salud a partir de la publicidad de medicamentos realizada por la industria. En cambio se encuentra reiteradamente documentada la influencia negativa de la misma en la relación médico paciente y en los costos de salud, tanto para los pacientes como para los sistemas de atención, lo que genera frecuentemente prácticas iatrogénicas ${ }^{9}$.

Debemos evitar caer en la medicina basada en la evidencia del marketing y preservar la medicina basada en la evidencia científica. La evidencia científica nos muestra la necesidad de regular un mercado imperfecto y mejorar el acceso a la información de la población general y de los pacientes en particular con el objetivo de que se encuentren en una situación que les permita tomar las 
mejores decisiones sobre su salud. Por otro lado, en este contexto de tamaña inequidad que estamos viviendo, es preocupante la generación masiva de necesidades espurias sobre tecnologías sanitarias sin evidencia científica que las respalde en un gran sector de la población que nunca podrá acceder a ellas. Ya bastante tienen con sus necesidades no espurias...

Carlos Silva [ Médico Especialista en Psiquiatría y Diplomado en Salud Pública. Equipo Psy Adrogué. ]

\section{Referencias}

1. Gilbody S, Wilson P, Watt I. Benefits and harms of direct to consumer advertising: a systematic review. Qual Saf Health Care. 2005 Aug;14(4):246-50.

2. Kravitz RL, Bell RA, Franz CE, Elliott MN, Amsterdam E, Willis C, Silverio L. Characterizing patient requests and physician responses in office practice. Health Serv Res. 2002 Feb;37(1):217-38.

3. Mintzes B. Direct to consumer advertising is medicalising normal human experiences. BMJ. 2002; 324:908-911

4. Bell RA, Wilkess MS, Kravitz RL. Advertisement-induced prescription drug request: patients anticipated reactions to a physician who refuses. J Fam Pract. $1999 ; 48(6): 446-52$.

5. Kravitz RL, Epstein R, Feldman MD, Franz CE, Azari R, Wilkes MS, Hinton L, Franks P. Influence of patients' requests for direct-to-consumer advertised antidepressants: a randomized controlled trial. JAMA. 2005; 293:16, 1995-2002.

6. Mintzes B, Barer ML, Kravitz RL, et al. Influence of DTC pharmaceutical advertising and patients' requests on prescribing decisions: two site cross sectional survey. BMJ. 2002; 324:278279 .

7. Wilkes MS, Bell RA, Kravitz RL. Direct-to-consumer prescription drug advertising: trends, impact, and implications. Health Aff (Millwood). 2000 Mar-Apr;19(2):110-28. Review. PMID: 10718026 disponible on line en http://content.healthaffairs.org/cgi/reprint/19/2/110

8. ANMAT, Argentina. Disponible en http://www.anmat.gov.ar/normativa/Normativa/NormasGenerales/Resolucion_20-2005.pdf

9. Gilbody S, Wilson P, Watt I. Benefits and harms of direct to consumer advertising: a systematic review. Qual Saf Health Care. 2005 Aug;14(4):246-50.

\section{Influencia prescriptiva de la solicitud de antidepresivos publicitados directamente a los consumidores}

Kravitz, et al. JAMA. 2005; 293:16.

\section{Objetivo}

Determinar el efecto de la publicidad directa al consumidor sobre el tratamiento inicial de pacientes con síntomas depresivos por médicos generalistas.

\section{Diseño}

Estudio aleatorizado utilizando actrices entrenadas como pacientes estandarizadas (PE). Se plantearon seis opciones, cruzando dos condiciones clínicas (depresión mayor o trastorno del estado de ánimo) con tres peticiones de tratamiento por parte de los PE (nombre comercial de un antidepresivo, un antidepresivo sin especificar cual o ninguna). Ese año el laboratorio Glaxo SmithKline había promocionado su antidepresivo Paxil ${ }^{\circledR}$ profusamente en avisos televisivos y gráficos.

\section{Lugar}

Consultorios de atención primaria en San Francisco y Nueva York, EEUU. Fue realizado entre 2003 y 2005.

\section{Participantes}

Participaron 152 médicos de familia o internistas de medicina general que trabajaban para Organizaciones de Atención Médica Gerenciada (HMO). Aceptaron participan entre $53 \%$ a $61 \%$ de los invitados.

\section{Intervención}

Los PE fueron asignados aleatoriamente para realizar 298 visitas de incógnito. Los médicos habían dado su consentimiento por escrito para recibir pacientes simulados a lo largo del año del estudio. A los médicos participantes se les había informado que recibirían dos visitas de pacientes simulados con síntomas comunes de la práctica clínica (no se les había detallado que tipo de síntomas) en meses diferentes. Cada médico recibió dos visitas a lo largo del año. Este antidepresivo no tenía genérico, pero era aceptado por los sistemas de salud y los médicos contaban con la alternativa de prescribir un preparado genérico de fluoxetina. EI protocolo fue aprobado por los comités de ética.

\section{Medición de los resultados principales}

Los datos obtenidos de las prescripciones, los reportes de los PE, el análisis de las grabaciones y los informes de los médicos. Para el análisis se utilizaron tablas de contingencia y confirmada ajustando por lugar de procedencia, médicos, y características de las visitas.

\section{Resultados principales}

El modelo utilizado permitió un muy bajo nivel de sospecha por parte de los médicos (13\%). Las diferencias en las conductas fueron significativamente diferentes en las dos condiciones clínicas. Los resultados se resumen en la tabla 1.

Tabla 1. Conducta prescriptiva de los médicos según la petición de las pacientes estandarizadas.

\begin{tabular}{|c|c|c|c|c|c|c|c|c|}
\hline & \multirow{2}{*}{$\begin{array}{l}\text { Tipo de } \\
\text { pedido }\end{array}$} & \multirow[t]{2}{*}{ Visitas } & \multicolumn{3}{|c|}{ Recilie nombre comercial } & \multicolumn{3}{|c|}{ Recibe nombre comercial } \\
\hline & & & n & $\%$ & IC $95 \%$ & $\mathbf{n}$ & $\%$ & CF $95 \%$ \\
\hline \multirow{3}{*}{$\begin{array}{l}\text { Episodio depresivo } \\
\text { mayor }\end{array}$} & Nombre comercial & 51 & 27 & 53 & $38-67$ & 14 & 27 & $16-42$ \\
\hline & General & 50 & 38 & 76 & $62-87$ & 1 & 2 & $0-11$ \\
\hline & Ninguno & 48 & 15 & 31 & $19-46$ & 2 & 4 & $0-14$ \\
\hline \multirow{3}{*}{$\begin{array}{l}\text { Trastorno del estado } \\
\text { de ánimo }\end{array}$} & Nombre comercial & 49 & 27 & 55 & $40-69$ & 18 & 37 & $23-52$ \\
\hline & General & 49 & 19 & 39 & $25-54$ & 5 & 10 & $3-22$ \\
\hline & Ninguno & 51 & 5 & 10 & $3 \cdot 21$ & 0 & 0 & 0.7 \\
\hline
\end{tabular}

\section{Conclusiones}

El requerimiento de los pacientes tiene una importante influencia sobre la conducta prescriptiva de los médicos generales en depresión mayor y trastornos del estado de ánimo. La publicidad directa al consumidor, puede generar sobremedicación, pero al mismo tiempo puede constituir una importante herramienta para comprometer a los pacientes en el cuidado de la depresión y probablemente en las enfermedades crónicas en general.

Fuente de financiamiento: Fuente de financiamiento: Instituto Nacional de Salud Mental (NIMH) de los Estados Unidos de Norteamérica. La agencia financiadota no participó del diseño, ejecución, análisis de datos ni interpretación de los resultados ni en la aprobación del manuscrito.

Resumido por Silva C de: Kravitz RL, Epstein R, Feldman MD, Franz CE, Azari R, Wilkes MS, Hinton L, Franks P. Influence of patients' requests for direct-toconsumer advertised antidepressants: a randomized controlled trial. JAMA. 2005; 293:16, 1995-2002. PMID: 15855433.

Referencias

1. Bell RA, Wilkess MS, Kravitz RL. Advertisement-induced prescription drug request: patients anticipated reactions to a physician who refuses. J Fam Pract. $1999 ; 48(6): 446-52$. 2. Kravitz RL, Bell RA, Franz CE, Elliott MN, Amsterdam E, Willis C, Silverio L. Characterizing patient requests and physician responses in office practice. Health Serv Res. 2002 Feb;37(1):217-38.

3. Mintzes B, Barer ML, Kravitz RL, et al. Influence of DTC pharmaceutical advertising and patients' requests on prescribing decisions: two site cross sectional survey. BMJ. 2002; 324:278279

4. Mintzes B. Direct to consumer advertising is medicalising normal human experiences. BMJ. 2002; 324:908-911.

5. Wilkes MS, Bell RA, Kravitz RL. Direct-to-consumer prescription drug advertising: trends, impact, and implications. Health Aff (Millwood). 2000 Mar-Apr;19(2):110-28. Review. PMID: 10718026 disponible on line en http://content.healthaffairs.org/cgi/reprint/19/2/110 